\title{
A finite multiplicity Helson-Lowdenslager-de Branges theorem
}

\author{
by
Sneh Lata (Houston, TX), Meghna Mittal (Houston, TX) and Dinesh Singh (Delhi)

Dedicated to the memory of Henry Helson

\begin{abstract}
We prove two theorems. The first theorem reduces to a scalar situation the well known vector-valued generalization of the Helson-Lowdenslager theorem that characterizes the invariant subspaces of the operator of multiplication by the coordinate function $z$ on the vector-valued Lebesgue space $L^{2}\left(\mathbb{T} ; \mathbb{C}^{n}\right)$. Our approach allows us to prove an equivalent version of the vector-valued Helson-Lowdenslager theorem in a completely scalar setting, thereby eliminating the use of range functions and partial isometries. The other three major advantages provided by our characterization are: (i) we provide precise necessary and sufficient conditions for the presence of reducing subspaces inside simply invariant subspaces; (ii) we give a complete description of the wandering vectors; (iii) we prove the theorem in the setting of all the Lebesgue spaces $L^{p}(0<p \leq \infty)$. Our second theorem generalizes the first theorem along the lines of de Branges' generalization of Beurling's theorem by characterizing those Hilbert spaces that are simply invariant under multiplication by $z^{n}$ and which are contractively contained in $L^{p}(1 \leq p \leq \infty)$. This also generalizes a theorem of Paulsen and Singh [Proc. Amer. Math. Soc. 129 (2000)] as well as the main theorem of Redett [Bull. London Math. Soc. 37 (2005)].
\end{abstract}

1. Introduction. The Helson-Lowdenslager invariant subspace theorem, $[\mathrm{HL}$, describes the simply invariant subspaces of the operator $S$ of multiplication by the coordinate function $z$ on $L^{2}$, thereby generalizing in a highly non-trivial fashion Beurling's famous invariant subspace theorem [Beu]. The theorem and the method invented by Helson and Lowdenslager are important for a variety of reasons and we refer to [DPRS], Gam, Hel1, [Hof2, [PS1, [PS2] for the details. The doubly invariant subspaces of $S$ are described by Wiener's theorem. We refer to [Hel2] for details. Between them, these two theorems describe the class of all invariant sub-

2010 Mathematics Subject Classification: Primary 47A15.

Key words and phrases: invariant subspaces, Helson-Lowdenslager theorem, de Branges theorem, Lebesgue spaces, finite multiplicity, shift part, multiplication by $z^{n}$. 
spaces for $S$ on scalar-valued $L^{2}$ of the unit circle. The description of such invariant subspaces of $S$ has been generalized and extended in many directions; we refer to [Gam, Hel2, [Hel3], [PS1], Red], [RaRo]. We also refer to Sin for several other useful references. Among these fruitful and interesting generalizations is the extension of these theorems to the context of functions on the unit circle taking values in an arbitrary Hilbert space $\mathcal{H}$. Such a generalization can be found among other places in Helson's book [Hel2] and in [RaRo, and also in $\mathrm{Ohn}$, [San], [Sri]. The tenor of these generalizations involves the description of the decomposition of these invariant subspaces into the direct sum of two invariant subspaces one of which is the shift part and the other is doubly invariant. Unlike the scalar case, such generalizations do point out that the two subspaces, the shift part and the doubly invariant part, can coexist to add up to the entire invariant subspace which remains simply invariant.

The purpose of this paper is to prove two theorems. The first of these looks anew at the solved problem of characterizing the invariant subspaces of $S$ on the Lebesgue space $L^{2}$ whose functions take values in a finitedimensional Hilbert space $\mathcal{H}$. More precisely, unlike the previous characterizations, we characterize - without taking recourse to vectorial concepts such as a range function and without stepping outside the scalar situation - the most general form of an invariant subspace of the operator of multiplication by $z^{n}$ in the classical Lebesgue space $L^{p}, 0<p \leq \infty$, on the unit circle. This includes describing the precise nature of the shift part of a simply invariant subspace by characterizing in an explicit and simple form the nature of its wandering vectors and by giving exact conditions under which a simply invariant subspace cannot contain a reducing subspace. We also establish when the reducing part of a simply invariant subspace is not zero and in that event we describe the precise nature of the reducing part in terms of the wandering vectors of the shift part.

The second theorem of this paper characterizes the class of all Hilbert spaces that are contractively contained in $L^{q}, 1 \leq q \leq 2$, and on which the operator of multiplication by $z^{n}$ acts isometrically. By imposing a natural condition, we give a precise description of such spaces, which also generalizes our first theorem as well as the main theorems in [PS1] and [Red]. This result is also relevant in the context of de Branges' generalization of Beurling's theorem (see [SS]), since our result can be viewed as the analogue of de Branges' theorem for the Helson-Lowdenslager theorem. This generalization of de Branges is the starting point of the theory of de Branges spaces. Some relevant references for de Branges spaces are [BR, Sar1, Sar2] and [SS.

This paper is organized as follows. In Section 2, we give some basic notations and definitions and the statement of the main results. In Section 3, 
we prove the preliminary results as well as the first main theorem. Section 4 deals with some basic results concerning the spaces that are contractively contained in $L^{p}$ and simply invariant under $S^{n}$, which leads us to the proof of the second main theorem. Also, we prove a direct generalization of the characterization, as given in [PS1], [Red], of those Hilbert spaces which are boundedly contained in $L^{p}$ and doubly invariant under $S^{n}$. Of course, while we deal with the case of a general positive integer $n$, those papers deal with $n=1$ only. Finally towards the end of Section 4 we give some final remarks and consequences of the results proved earlier.

\section{Notations, terminology and statement of the main results.}

We denote the unit circle in the complex plane by $\mathbb{T}$ and the open unit disk by $\mathbb{D}$. For any $0<p \leq \infty, L^{p}$ and $H^{p}$ denote the familiar Lebesgue and Hardy spaces respectively on $\mathbb{T}$. Recall that $L^{p}$, and consequently also $H^{p}$, are Banach spaces with the usual norm $\|f\|_{p}=\left((2 \pi)^{-1} \int_{0}^{2 \pi}|f|^{p} d m\right)^{1 / p}$ when $1 \leq p<\infty$ and $\|f\|_{\infty}=\operatorname{ess} \sup |f|$ when $p=\infty$. In particular, when $p=2$ these are Hilbert spaces with the inner product $\langle f, g\rangle=(2 \pi)^{-1} \int_{0}^{2 \pi} f \bar{g} d m$. When $0<p<1, L^{p}$ and $H^{p}$ are complete metric spaces with respect to the metric $d(f, g)=\|f-g\|_{p}^{p}=(2 \pi)^{-1} \int_{0}^{2 \pi}|f-g|^{p} d m$. For a fixed positive integer $n, L^{p}\left(z^{n}\right)$ denotes the closed linear span of $\left\{z^{k n}: k \in \mathbb{Z}\right\}$ in $L^{p}$ where $z$ is the coordinate function on $\mathbb{T}$, and $H^{p}\left(z^{n}\right)$ stands for $L^{p}\left(z^{n}\right) \cap H^{p}$.

A Hilbert space $\mathcal{M}$ is said to be boundedly contained in a Banach space $\mathcal{B}$ if $\mathcal{M}$ is a vector subspace of $\mathcal{B}$ and the inclusion map is bounded, i.e. $\|x\|_{\mathcal{B}} \leq C\|x\|_{\mathcal{M}}$ for all $x$ in $\mathcal{M}$ and some constant $C$. When $C=1$, we say that $\mathcal{M}$ is contractively contained in $\mathcal{B}$. For further details on all of the above we refer to [Hel1], [HL] and [Hof1].

For a fixed positive integer $n, S^{n}$ denotes the operator of multiplication by $z^{n}$. It is easy to see that $S^{n}$ is an isometry on $L^{2}$ and hence also on $H^{2}$. A closed subspace $\mathcal{M}$ of $L^{p}, 0<p \leq \infty$ ( weak $^{*}$-closed when $p=\infty$ ) is said to be simply invariant under $S^{n}$ if $S^{n}(\mathcal{M}) \subsetneq \mathcal{M}$, and doubly invariant if $S^{n}(\mathcal{M})=\mathcal{M}$. For our purposes, a Hilbert space $\mathcal{M}$ which is boundedly contained in $L^{p}$ will be said to be simply [doubly] invariant if $S^{n}(\mathcal{M}) \subsetneq \mathcal{M}$ $\left[S^{n}(\mathcal{M})=\mathcal{M}\right]$.

If $T$ is a bounded operator from a Hilbert space $\mathcal{K}$ into $L^{p}$, then we let $\mathcal{R}(T)$ denote the range of $T$, which is a (not necessarily closed) subspace of $L^{p}$. However, if we endow $\mathcal{R}(T)$ with the norm $\|h\|_{\mathcal{R}(T)}=\inf \left\{\|k\|_{\mathcal{K}}\right.$ : $T k=h\}$, then $\mathcal{R}(T)$ is a Hilbert space in this norm, called the range space of $T$, and it is boundedly contained in $L^{p}$. When $T$ is a contraction then the range space of $T$ is contractively contained in $L^{p}$.

Before proceeding with some more terminology we note that we can write

$$
L^{p}=L^{p}\left(z^{n}\right) \oplus z L^{p}\left(z^{n}\right) \oplus \cdots \oplus z^{n-1} L^{p}\left(z^{n}\right) .
$$


Obviously, by the above direct sum we mean the Hilbert space direct sum when $p=2$ and the algebraic direct sum otherwise.

The following definition, concerning $L^{\infty}$ functions, is a direct analogue of the definition of $n$-inner functions in $H^{\infty}$, introduced by the third author and Thukral in $[\mathrm{ST}]$.

Definition 2.1. A function $\phi \in L^{\infty}$ is called $n$-unimodular if $\sum_{i=1}^{n}\left|\phi_{i}\right|^{2}$ $=1$ a.e., where $\phi=\sum_{i=1}^{n} z^{i-1} \phi_{i}$ with $\phi_{i} \in L^{\infty}\left(z^{n}\right)$.

Lastly, we define an operator on $L^{2}$ which is an analogue of the multiplication operator, usually denoted by $M_{\phi}$ where $\phi \in L^{\infty}$. Take a matrix $\psi=\left(\psi_{i j}\right) \in M_{m n}\left(L^{\infty}\right)$ and define an operator $A_{\psi}$ on $L^{2}$ by

$$
A_{\psi}(f)=\sum_{i=1}^{m} z^{i-1}\left(\sum_{j=1}^{n} \psi_{i j} f_{j}\right)
$$

where $f=\sum_{i=1}^{n} z^{i-1} f_{i}$ with $f_{i} \in L^{2}\left(z^{n}\right)$. It is easy to see that $A_{\psi}$ is a bounded operator on $L^{2}$ and it reduces to the usual multiplication operator on $L^{2}$ when $n=m=1$. Note that the kernel of $A_{\psi}$ is a doubly invariant subspace of $L^{2}$ under $S^{n}$. We write $\mathcal{K}_{\mathcal{M}}^{\psi}$ for the kernel of $A_{\psi}$ restricted to the subspace $\mathcal{M}$ of $L^{2}$.

By $\bar{\psi}$ we mean the matrix $\left(\overline{\psi_{i j}}\right)$, where $\overline{\psi_{i j}}$ is the complex conjugate of the function $\psi_{i j}$.

For our purposes, $\mathcal{K}_{\mathcal{M}}^{\psi}$ is specific in nature. To see this, let $\left\{\psi_{1}, \ldots, \psi_{r}\right\}$ be a set of $n$-unimodular functions in $L^{\infty}$ and let $\psi=\left(\psi_{i j}\right)$, where $\psi_{i}=$ $\sum_{j=1}^{n} z^{j-1} \psi_{i j}, \psi_{i j} \in L^{\infty}\left(z^{n}\right)$. Then it can be easily seen that

$$
\mathcal{K}_{\mathcal{M}}^{\psi}=\left\{f \in \mathcal{M}: \sum_{j=1}^{n} \psi_{i j} f_{j}=0 \text { a.e. } \forall 1 \leq i \leq r\right\},
$$

where $f=\sum_{j=1}^{n} z^{j-1} f_{j}, f_{j} \in L^{2}\left(z^{n}\right)$. We can use this to verify that $\mathcal{K}_{L^{2}}^{\bar{\psi}}$ is the maximal doubly invariant subspace of $L^{2}$ under $S^{n}$ that is orthogonal to $\psi_{i} H^{2}\left(z^{n}\right)$ for all $1 \leq i \leq n$.

Keeping this motivation in mind, we define, for any subspace $\mathcal{M}$ of $L^{p}$,

$$
\mathcal{K}_{\mathcal{M}}^{\psi}=\left\{f \in \mathcal{M}: \sum_{j=1}^{n} \psi_{i j} f_{j}=0 \text { a.e. } \forall 1 \leq i \leq r\right\},
$$

where $f=\sum_{j=1}^{n} z^{j-1} f_{j}, f_{j} \in L^{p}\left(z^{n}\right)$.

We are now in a position to state our main results.

Theorem A. Let $\mathcal{M}$ be a subspace of $L^{p}, 0<p \leq \infty$, simply invariant under $S^{n}$. Then the most general form that $\mathcal{M}$ can assume is

$$
\mathcal{M}=\sum_{i=1}^{r} \oplus \phi_{i} H^{p}\left(z^{n}\right) \oplus \mathcal{K}_{\mathcal{M}}^{\bar{\phi}}
$$


where

(i) $r \leq n$,

(ii) each $\phi_{i}$ is an n-unimodular function not vanishing on any set of positive measure,

(iii) $\left\|\phi_{i} f\right\|_{2}=\|f\|_{2}$ for every $f \in L^{2}\left(z^{n}\right)$,

(iv) $\phi=\left(\phi_{i j}\right) \in M_{n}\left(L^{\infty}\left(z^{n}\right)\right), \phi_{i}=\sum_{j=1}^{n} z^{j-1} \phi_{i j}$.

Moreover, if $r=n$, then $\mathcal{K}_{\mathcal{M}}^{\bar{\phi}}=\{0\}$, and if $r<n$, then there exist infinitely many non-zero doubly invariant subspaces of $\mathcal{K}_{L^{2}}^{\bar{\phi}}$ which when appended to $\sum_{i=1}^{r} \oplus \phi_{i} H^{2}\left(z^{n}\right)$ form a simply invariant subspace of $L^{2}$.

In [PS1, the authors prove that the straightforward generalization of the Helson-Lowdenslager theorem along the lines of de Branges' generalization of Beurling's theorem is not possible, by constructing an example of a subspace $\mathcal{M}$ of $L^{2}$ which is simply invariant under $S$; this justifies the requirement of an extra condition that appears in (1) below. In addition, the condition is natural in the sense that in the simplest situation when $\mathcal{M}$ is a closed subspace of $L^{q},\|f\|_{q} \leq\|f\|_{p}$ for all $f \in \mathcal{M}$ whenever $p>q$.

The second of our two main theorems shows that under similar conditions to those considered in [PS1] and [Red, there are non-trivial Hilbert spaces contractively contained in $L^{p}$ for $1 \leq p \leq 2$. The analogous result for the case $2<p \leq \infty$ to the one obtained by the third author and Paulsen in [PS1] can be found in a later section; it asserts that no non-trivial simply invariant Hilbert space under $S^{n}$ is contractively contained in $L^{p}$. We shall adopt the notation $2 q /(2-q)=\infty$ when $q=2$.

Theorem B. Let $\mathcal{M} \neq 0$ be a simply invariant Hilbert space contractively contained in $L^{q}, 1 \leq q \leq 2$, and on which $S^{n}$ acts isometrically. Further, suppose that there exists $p$ with $2 \leq p \leq 2 q /(2-q)$ and $a \delta>0$ such that

$$
\|f\|_{\mathcal{M}} \leq \delta\|f\|_{p} \quad \forall f \in \mathcal{M} \cap L^{p} .
$$

Then there exists an orthonormal set $\left\{\psi_{i}\right\}_{i=1}^{r} \subseteq \mathcal{M} \cap L^{\infty}, r \leq n$, with each element non-zero a.e. such that

$$
\mathcal{M}=\sum_{i=1}^{r} \oplus \psi_{i} H^{2}\left(z^{n}\right) \oplus \mathcal{R}\left(M_{\mu} A_{\phi}\right),
$$

where

(i) $\phi \in M_{n}\left(L^{\infty}\left(z^{n}\right)\right)$ and $\mu \in L^{2 q /(2-q)}$ is a strictly positive function; in particular, $\mu=1$ a.e. when $q=2$,

(ii) $\left\|\psi_{i} f\right\|_{\mathcal{M}}=\|f\|_{2}$ for every $f \in H^{2}\left(z^{n}\right)$ and for every $1 \leq i \leq r$, 
(iii) $\left(\sum_{j=1}^{r}\left|\psi_{i j}\right|\right)^{-1} \in L^{s}$ and $\left\|\left(\sum_{j=1}^{r}\left|\psi_{i j}\right|\right)^{-1}\right\|_{s} \leq \delta$ where $\psi_{i}=$ $\sum_{j=1}^{r} z^{j-1} \psi_{i j}$ for $1 \leq i \leq r$, and

$$
s= \begin{cases}2 p /(p-2) & \text { if } 2<p \leq 2 q /(2-q), \\ \infty & \text { if } p=2\end{cases}
$$

Moreover, if $r=n$ then $\mathcal{R}\left(M_{\mu} A_{\phi}\right)=\{0\}$.

3. Proof of Theorem A. We begin with some preliminary results required for the proof of the first main theorem.

LEMMA 3.1. Let $\mathcal{M}$ be a subspace of $L^{2}$ which is simply invariant un$\operatorname{der} S^{n}$. Denote $\mathcal{N}=\mathcal{M} \ominus S^{n}(\mathcal{M})$. Then:

(i) $\left\{\phi z^{k n}\right\}_{k \in \mathbb{Z}}$ is an orthonormal set for each unit vector $\phi \in \mathcal{N}$. Consequently, $\|\phi f\|_{2}=\|f\|_{2}$ for every $f \in L^{2}\left(z^{n}\right)$.

(ii) Every unit vector $\phi \in \mathcal{N}$ is n-unimodular.

(iii) $\mathcal{N} \subseteq L^{\infty}$.

(iv) If $\{\phi, \psi\}$ is an orthogonal set in $\mathcal{N}$, then $\sum_{j=1}^{n} \phi_{j} \bar{\psi}_{j}=0$ a.e., where $\phi=\sum_{j=1}^{n} z^{j-1} \phi_{j}$ and $\psi=\sum_{j=1}^{n} z^{j-1} \psi_{j}$, with $\phi_{j}$ and $\psi_{j}$ in $L^{\infty}\left(z^{n}\right)$ for all $1 \leq j \leq n$.

(v) Elements of $\mathcal{N}$ cannot vanish on sets of positive measure.

Proof. By the Wold decomposition [Hof1, p. 109], we have

$$
\mathcal{M}=\sum_{k=0}^{\infty} \oplus S^{k n}(\mathcal{N}) \oplus \bigcap_{k \geq 0} S^{k n}(\mathcal{M}) .
$$

It then immediately follows that $\left\{\phi z^{k n}\right\}_{k \in \mathbb{Z}}$ is an orthonormal set for each unit vector $\phi \in \mathcal{N}$. To see the last statement in (i), let $f \in L^{2}\left(z^{n}\right)$ be such that $f_{l}=\sum_{k=-l}^{l} \alpha_{k} z^{k n}$ converges to $f$ in $L^{2}$. Then

$$
\left\|\phi f_{l}\right\|_{2}=\left\|\sum_{k=-l}^{l} \alpha_{k} \phi z^{k n}\right\|_{2}=\left(\sum_{k=-l}^{l}\left|\alpha_{k}\right|^{2}\right)^{1 / 2}=\left\|f_{l}\right\|_{2} .
$$

By a limiting argument, we see that $\|\phi f\|_{2}=\|f\|_{2}$ for every $f \in L^{2}\left(z^{n}\right)$. Note that (iii) follows from (ii). To prove (ii), let $\phi \in \mathcal{N}$ with $\|\phi\|_{2}=1$. Then by (i), $\phi \perp \phi z^{k n}$ for every $k \neq 0$. This yields $(2 \pi)^{-1} \int_{0}^{2 \pi}|\phi|^{2} z^{k n}=0$ for every $k \neq 0$. If we write $\phi=\sum_{j=1}^{n} z^{j-1} \phi_{j}$ with $\phi_{j} \in L^{2}\left(z^{n}\right)$ then $\sum_{j=1}^{n}\left|\phi_{j}\right|^{2}=\delta$ for some constant $\delta$. It is easy to see that $\delta=1$, since $\|\phi\|_{2}=1$. This completes the proof of (ii).

To prove (iv), let $\{\phi, \psi\}$ be an orthogonal set in $\mathcal{N}$. Then the Wold decomposition gives $\left\langle\phi, z^{k n} \psi\right\rangle=0$ for all $k \in \mathbb{Z}$. Let $\phi=\sum_{j=1}^{n} z^{j-1} \phi_{j}$ and $\psi=\sum_{j=1}^{n} z^{j-1} \psi_{j}$, where as usual $\phi_{j}, \psi_{j} \in L^{\infty}\left(z^{n}\right)$. Then the above yields $\left\langle\sum_{i=1}^{n} \phi_{i} \bar{\psi}_{i}, z^{k n}\right\rangle=0$ for all $k$. Since $\sum_{i=1}^{n} \phi_{i} \overline{\psi_{i}} \in L^{2}\left(z^{n}\right)$, it follows that $\sum_{i=1}^{n} \phi_{i} \overline{\psi_{i}}=0$ a.e. 
Finally we prove (v). Take a non-zero $\phi$ in $\mathcal{N}$ with $\|\phi\|_{2}=1$ and suppose towards a contradiction that there exists a set $A$ in $\mathbb{T}$ of positive measure such that $\phi$ vanishes on $A$. Define

$$
k_{m}= \begin{cases}m & \text { on } B \\ 1 & \text { on } B^{c}\end{cases}
$$

where $B=\left\{z^{n}: z \in A\right\}$. Note that $k_{m} \in L^{\infty}$ for each $m$. Let $t_{m}(z)=k_{m}\left(z^{n}\right)$ and $h_{m}=\exp \left(t_{m}+i \tilde{t}_{m}\right)$, where $\tilde{t}_{m}$ denotes the harmonic conjugate of $t_{m}$, so that $h_{m} \in H^{\infty}\left(z^{n}\right)$ for all $m$. Let $h_{m}=\sum_{k=0}^{\infty} \alpha_{k} z^{k n}$. Then by (i) we get

$$
\left\|\phi \sum_{k=0}^{l} \alpha_{k} z^{k n}\right\|_{2}=\left(\sum_{k=0}^{l}\left|\alpha_{k}\right|^{2}\right)^{1 / 2}=\left\|\sum_{k=0}^{l} \alpha_{k} z^{k n}\right\|_{2} .
$$

Note that $\phi \sum_{k=0}^{l} \alpha_{k} z^{k n}$ is in $\mathcal{M}$ and is Cauchy in $L^{2}$. This shows that $\phi h_{m} \in \mathcal{M}$ and $\left\|\phi h_{m}\right\|_{2}=\left\|h_{m}\right\|_{2}$. By the definition of $h_{m}$, we get $\left\|\phi h_{m}\right\|_{2}$ $<K$ for some constant $K$ independent of $m$ and $\left\|h_{m}\right\|_{2}>\sqrt{\exp (2 m) m(A)}$, which is indeed a contradiction. This completes the proof of the lemma.

LEMMA 3.2. Let $\left\{\phi_{1}, \ldots, \phi_{n}\right\}$ be a set of n-unimodular functions such that $\phi_{i} L^{2}\left(z^{n}\right)$ are pairwise orthogonal. Write $\phi_{i}(z)=\sum_{j=1}^{n} z^{j-1} \phi_{i j}(z)$ with $\phi_{i j} \in L^{2}\left(z^{n}\right)$ for every $i, j \in\{1, \ldots, n\}$, and define

$$
A_{r}(z)=\left(\begin{array}{ccc}
\phi_{11}(z) & \cdots & \phi_{1 n}(z) \\
\vdots & \vdots & \vdots \\
\phi_{r 1}(z) & \cdots & \phi_{r n}(z)
\end{array}\right)
$$

for every $1 \leq r \leq n$. Then $A_{r} \in B(\underbrace{L^{2}\left(z^{n}\right) \oplus \cdots \oplus L^{2}\left(z^{n}\right)}_{r \text { copies }})$, and $A_{n}(z)$ is unitary for almost all $z$. In particular, when $r<n$, then $A_{r}(z)$ is a coisometry for almost all $z$.

Proof. Since $\sum_{j=1}^{n}\left|\phi_{i j}\right|^{2}=1$ for every $1 \leq i \leq r$, it is easy to see that $A_{r}$ is a bounded operator on $\underbrace{L^{2}\left(z^{n}\right) \oplus \cdots \oplus L^{2}\left(z^{n}\right)}_{r \text { copies }}$. By using the same logic that is used to deduce Lemma 3.1(iv), we get $\sum_{l=1}^{n} \phi_{i l} \overline{\phi_{j l}}=0$ a.e. for all $i \neq j$. This together with the hypothesis that each $\phi_{i}$ is $n$-unimodular yields the required result.

REMARK 3.3. When $r<n$ in the above lemma, there exist infinitely many functions $f \in K_{L^{2}}^{A_{r}}$, that is, $f \in L^{2}$ such that $\sum_{j=1}^{n} \phi_{i j} f_{j}=0$ for every $1 \leq i \leq r$, where $f(z)=\sum_{j=1}^{n} z^{j-1} f_{j}(z)$ for almost all $z$.

We record an immediate corollary of the above lemma for our later use. 
COROLlary 3.4. Let $\left\{\phi_{1}, \ldots, \phi_{n}\right\}$ be a set of $n$-unimodular functions such that $\phi_{i} L^{2}\left(z^{n}\right)$ are pairwise orthogonal. Then there exists no non-zero $\psi \in L^{2}$ such that $\psi \perp \phi_{i} L^{2}\left(z^{n}\right)$ for each $i$.

LEMMA 3.5. Let $\left\{f_{k}\right\}$ be a sequence in $L^{2}$ that converges to some function $f$ in $L^{2}$. Then there exists a sequence $\left\{h_{k}\right\}$ in $H^{\infty}$, with $\left\|h_{k}\right\|_{\infty} \leq 1$ for all $k$, such that a subsequence of it converges to 1 a.e., and the sequence $\left\{h_{k} f_{k}\right\}$ is bounded above by an integrable function. Moreover, if $f_{k}-f \in L^{2}\left(z^{n}\right)$, then $h_{k} \in H^{\infty}\left(z^{n}\right)$.

Proof. Let $g_{k}=\left|f_{k}-f\right|$. Then $\left\{g_{k}\right\}$ is a sequence of real-valued functions in $L^{2}$ that converges to 0 in $L^{2}$, therefore there exists a harmonic conjugate $\tilde{g}_{k} \in L^{2}$ which also converges to 0 in $L^{2}$. For each $k$, define

$$
h_{k}=e^{-\left(g_{k}+\mathbf{i} \tilde{g}_{k}\right)} \text {. }
$$

Clearly, $h_{k} \in H^{\infty}$ and $\left\|h_{k}\right\|_{\infty} \leq 1$ for all $k$. We find a subsequence of $\left\{g_{k}\right\}$ and a subsequence of $\left\{\widetilde{g}_{k}\right\}$ such that both converge to 0 pointwise almost everywhere. It then follows that the corresponding subsequence of $\left\{h_{k}\right\}$ converges to 1 a.e. Now,

$$
\left|h_{k} f_{k}\right| \leq\left|h_{k}\left(f_{k}-f\right)\right|+\left|h_{k} f\right| \leq\left|h_{k} g_{k}\right|+|f| \leq 1+|f|,
$$

so $\left\{h_{k} f_{k}\right\}$ is bounded above by an integrable function. The last statement clearly follows from the construction of $h_{k}$.

Lemma 3.6. Let $\mathcal{M}$ be a simply invariant subspace of $L^{p}$ with $2<p \leq \infty$ under $S^{n}$. Then $\overline{\mathcal{M}}^{L^{2}} \cap L^{p}=\mathcal{M}$.

Proof. Let $g \in \overline{\mathcal{M}}^{L^{2}} \cap L^{p}$. Then there exists a sequence $\left\{f_{k}\right\}_{k} \subseteq \mathcal{M}$ such that $f_{k} \rightarrow g$ in $L^{2}$. Note that there exist $f_{k j}, g_{j} \in L^{p}\left(z^{n}\right)$ such that $f_{k}=\sum_{j=1}^{n} z^{j-1} f_{k j}, g=\sum_{j=1}^{n} z^{j-1} g_{j}$ and $f_{k j} \rightarrow g_{j}$ in $L^{2}$ for each $j$. By Lemma 3.5, there exists a sequence $\left\{h_{k j}\right\}$ in $H^{\infty}\left(z^{n}\right)$, with $\left\|h_{k j}\right\|_{\infty} \leq 1$ for all $k$, such that a subsequence of it converges to 1 a.e. and the sequence $\left\{h_{k j} f_{k j}\right\}$ satisfies $\left|h_{k j} f_{k j}\right| \leq 1+\left|g_{j}\right|$ for every $j$. To avoid too many subscripts, we may assume that $h_{k j}$ converges to 1 a.e., and that $f_{k j}$ converges to $g_{j}$ a.e., which further implies that $f_{k}$ converges to $g$ a.e.

Further, note that

$$
\left|f_{k} \prod_{i=1}^{n} h_{k i}\right|=\left|\sum_{j=1}^{n} z^{j-1} h_{k j} f_{k j} \prod_{i \neq j}^{n} h_{k i}\right| \leq \sum_{j=1}^{n}\left(1+\left|g_{j}\right|\right)
$$

where the function on the right hand side is in $L^{p}$. Thus, by the dominated convergence theorem, $f_{k} \prod_{j=1}^{n} h_{k j} \rightarrow g$ in $L^{p}$ for $p<\infty$ and $f_{k}\left(\prod_{j=1}^{n} h_{k j}\right) \rightarrow g$ in the weak* topology for $p=\infty$. Lastly, the fact that $\prod_{j=1}^{n} h_{k j}$ is a sequence in $H^{\infty}\left(z^{n}\right)$ and $\mathcal{M}$ is a simply invariant subspace of $L^{p}$ implies that $f_{k}\left(\prod_{j=1}^{n} h_{k j}\right) \in \mathcal{M}$ and hence $g \in \mathcal{M}$. 
REMARK 3.7. The above lemma shows that no proper $S^{n}$-invariant subspace of $L^{p}, 2<p \leq \infty$, is dense in $L^{2}$.

Lemma 3.8. Let $f \in L^{2}$ with a decomposition $f=\sum_{k=1}^{r} \phi_{k} h_{k}+s$, where

(i) $r \leq n$,

(ii) $\left\{\phi_{k}\right\}_{k=1}^{r}$ is an orthonormal set in $L^{2}$,

(iii) $h_{k} \in H^{2}\left(z^{n}\right)$ for each $k, 1 \leq k \leq r$,

(iv) $s$ is orthogonal to $\sum_{k=1}^{r} \phi_{k} h_{k}$.

Then

$$
\sum_{k=1}^{r}\left|h_{k}(z)\right|^{2}+\sum_{l=1}^{n}\left|s_{l}(z)\right|^{2}=\frac{\sum_{j=0}^{n-1}\left|f\left(\alpha^{j} z\right)\right|^{2}}{n} \leq \frac{\left(\sum_{j=0}^{n-1}\left|f\left(\alpha^{j} z\right)\right|\right)^{2}}{n},
$$

where $\alpha=e^{2 \pi i / n}$ and $s=\sum_{l=1}^{n} z^{l-1} s_{l}$ with $s_{l} \in L^{2}\left(z^{n}\right)$.

Proof. Note that for each $k$, we can write $\phi_{k}=\sum_{m=1}^{n} z^{m-1} \phi_{k m}$ with $\phi_{k m} \in L^{2}\left(z^{n}\right)$ for every $m$. Then

$$
\begin{aligned}
\sum_{j=0}^{n-1}\left|f\left(\alpha^{j} z\right)\right|^{2}= & \sum_{j=0}^{n-1}\left|\sum_{k=1}^{r} \phi_{k}\left(\alpha^{j} z\right) h_{k}(z)+s\left(\alpha^{j} z\right)\right|^{2} \\
= & \sum_{j=0}^{n-1}\left|\sum_{m=1}^{n} \alpha^{j(m-1)} z^{m-1}\left(\sum_{k=1}^{r} \phi_{k m}(z) h_{k}(z)+s_{m}(z)\right)\right|^{2} \\
= & \sum_{j=0}^{n-1} \sum_{m, l=1}^{n} \alpha^{j(m-l)} z^{m-l}\left(\sum_{k=1}^{r} \phi_{k m}(z) h_{k}(z)+s_{m}(z)\right) \\
& \times\left(\sum_{k=1}^{r} \phi_{k m}(z) h_{k}(z)+s_{m}(z)\right) \\
= & \sum_{m=1}^{n} n\left|\sum_{k=1}^{r} \phi_{k m}(z) h_{k}(z)+s_{m}(z)\right|^{2} \\
= & n\left(\sum_{k=1}^{r}\left|h_{k}(z)\right|^{2}+\sum_{l=1}^{n}\left|s_{l}(z)\right|^{2}\right) .
\end{aligned}
$$

where the last equality follows by using hypotheses (ii) and (iv).

Finally, the asserted inequality follows from a simple algebra of positive numbers.

We are now in a position to prove the first of our two main theorems.

Proof of Theorem A. We split the proof into three cases.

Case (1). Assume $p=2$. By using the Wold decomposition, we get

$$
\mathcal{M}=\mathcal{M}_{1} \oplus \mathcal{K}
$$


where $\mathcal{K}=\bigcap_{k \geq 0} S^{n k}(\mathcal{M}), \mathcal{M}_{1}=\sum_{k=0}^{\infty} \oplus S^{k n}(\mathcal{N})$ and $\mathcal{N}=\mathcal{M} \ominus S^{n}(\mathcal{M})$. Clearly, $\bigcap_{k \geq 0} S^{k n}\left(\mathcal{M}_{1}\right)=\{0\}$ and $\bigcap_{k \geq 0} S^{k n}(\mathcal{K})=\mathcal{K}$. To establish the result, we claim that the dimension of $\mathcal{N}$ is at most $n$.

Suppose there exists an orthonormal set $\left\{\phi_{1}, \ldots, \phi_{n+1}\right\}$ in $\mathcal{N}$. Then by Lemma 3.1(ii)\&(iv) each $\phi_{i}$ is $n$-unimodular and $\phi_{n+1} \perp \phi_{i} L^{2}\left(z^{n}\right)$ for every $1 \leq i \leq n$. Thus, by Corollary 3.4, it follows that $\phi_{n+1}=0$, which contradicts the fact that $\left\|\phi_{n+1}\right\|=1$. This proves that the dimension of $\mathcal{N}$ is at most $n$ and hence

$$
\mathcal{M}_{1}=\sum_{i=1}^{r} \oplus \phi_{i} H^{2}\left(z^{n}\right)
$$

where $r \leq n$, and $\left\{\phi_{1}, \ldots, \phi_{r}\right\}$ is an orthonormal basis of $\mathcal{N}$. The rest of the properties of $\left\{\phi_{i}\right\}_{i=1}^{r}$ follow from Lemma 3.1.

Lastly, we need to see that $\mathcal{K}=\mathcal{K}_{\mathcal{M}}^{\bar{\phi}}$. If we let $f \in \mathcal{K}_{\mathcal{M}}^{\bar{\phi}}$, then $f \in \mathcal{M}$ and $\sum_{j=1}^{n} f_{j} \overline{\phi_{i j}}=0$ for all $i$, where $f=\sum_{j=1}^{n} z^{j-1} f_{j}, f_{i} \in L^{2}\left(z^{n}\right)$ and $\phi_{i}=\sum_{j=1}^{n} \phi_{i j}, \phi_{i j} \in L^{2}\left(z^{n}\right)$. Thus, for each $i,\left\langle f, z^{l n} \phi_{i}\right\rangle=0$ for all $l \geq 0$. This proves that $f$ is orthogonal to $\sum_{i=1}^{r} \oplus \phi_{i} H^{2}\left(z^{n}\right)$ in $\mathcal{M}$, and hence $\mathcal{K}_{\mathcal{M}}^{\bar{\phi}} \subseteq \mathcal{K}$.

To prove the other containment, we let $f \in \mathcal{K}$. We need to show that $\sum_{j=1}^{n} f_{j} \overline{\phi_{i j}}=0$ for all $i$. Note that $\sum_{j=1}^{n} f_{j} \overline{\phi_{i j}}$ is in $L^{2}\left(z^{n}\right)$, thus it is enough to show that it is orthogonal to $z^{l n}$ for every integer $l$. Indeed,

$$
\begin{aligned}
\left\langle\sum_{j=1}^{n} f_{j} \overline{\phi_{i j}}, z^{l n}\right\rangle & =\sum_{j=1}^{n}\left\langle z^{-l n} f_{j}, \phi_{i j}\right\rangle=\left\langle\sum_{j=1}^{n} z^{j-1} z^{-l n} f_{j}, \sum_{j=1}^{n} z^{j-1} \phi_{i j}\right\rangle \\
& =\left\langle z^{-l n} f, \phi_{i}\right\rangle=0,
\end{aligned}
$$

where the last equality follows from the fact that $S^{n}$ acts unitarily on $\mathcal{K}$ and $\phi_{i}$ 's are orthogonal to $\mathcal{K}$. Hence, $\sum_{j=1}^{n} f_{j} \overline{\phi_{i j}}=0$, and thereby $f \in \mathcal{K}_{\mathcal{M}}^{\bar{\phi}}$. Thus, we get $\mathcal{K}=\mathcal{K}_{\mathcal{M}}^{\bar{\phi}}$.

Finally, if $r=n$ then by Lemma 3.2 we see that $\mathcal{K}_{\mathcal{M}}^{\bar{\phi}}=\{0\}$, and if $r<n$ then by Remark 3.3 we find that there exist infinitely many functions in $\mathcal{K}_{L^{2}}^{\bar{\phi}}$. Consequently, we get infinitely many non-zero subspaces $\mathcal{M}^{\prime}$ of $L^{2}$, which gives rise to infinitely many non-zero doubly invariant subspaces $\mathcal{K}_{\mathcal{M}^{\prime}}^{\bar{\phi}} \subseteq \mathcal{K}_{L^{2}}^{\bar{\phi}}$ such that $\sum_{i=1}^{r} \oplus \phi_{i} H^{2}\left(z^{n}\right) \oplus \mathcal{K}_{\mathcal{M}^{\prime}}^{\bar{\phi}}$ is a simply invariant subspace of $L^{2}$. This completes the proof of the case $p=2$.

Case (2). Assume $0<p<2$. We first claim that

$$
\mathcal{M} \cap L^{2} \neq 0 \text {. }
$$


Let $f \in \mathcal{M}$. Then $|f|^{p / 2}$ is in $L^{2}$. It is easy to see that

$$
g(z)=\frac{\sum_{i=0}^{n-1}|f|^{p / 2}\left(\alpha^{i} z\right)}{n}
$$

belongs to $L^{2}\left(z^{n}\right)$, where $\alpha=e^{2 \pi i / n}$. If $h(z)$ denotes the harmonic conjugate of $g(z)$, then $h \in L^{2}\left(z^{n}\right)$ and hence $g+i h$ is in $H^{2}\left(z^{n}\right)$. If we set $k=$ $\exp [-(g+i h)]$, then $k$ is an outer function in $H^{\infty}\left(z^{n}\right)$. It is immediate to see that $k f \in L^{\infty}$ and hence in $L^{2}$. To see that $k f \in \mathcal{M}$, let $k_{m}$ be the $m$ th Cesàro mean of $k$. Then each $k_{m}$ is a polynomial in $H^{2}\left(z^{n}\right)$ and so $k_{m} f$ is in $\mathcal{M}$ for every $m$. Further, $\left\|k_{m}\right\|_{\infty} \leq\|k\|_{\infty}$ and $k_{m} \rightarrow k$ almost everywhere. Thus $\left\|k_{m} f-k f\right\|_{p} \rightarrow 0$. This shows that $k f$ is in $\mathcal{M}$ and thus $\mathcal{M} \cap L^{2}$ is a non-trivial subspace of $L^{2}$.

Note that $\mathcal{M} \cap L^{2}$ is closed in $L^{2}$ because $\mathcal{M}$ is closed in $L^{p}$. Also, $\mathcal{M} \cap L^{2}$ is a simply invariant subspace of $L^{2}$ under $S^{n}$, so by Case (1) we conclude that

$$
\mathcal{M} \cap L^{2}=\sum_{i=1}^{r} \oplus \phi_{i} H^{2}\left(z^{n}\right) \oplus \mathcal{K}_{\mathcal{M} \cap L^{2}}^{\bar{\phi}}
$$

where $\left\{\phi_{i}\right\}_{i=1}^{r}$ is a set of $n$-unimodular orthonormal vectors that do not vanish on any set of positive measure and $\phi$ is the corresponding matrix in $L^{\infty}\left(z^{n}\right)$ as obtained in Case (1).

We claim that

$$
\mathcal{M}=\sum_{i=1}^{r} \oplus \phi_{i} H^{p}\left(z^{n}\right) \oplus \mathcal{K}_{\mathcal{M}}^{\bar{\phi}}
$$

Since $\mathcal{M}$ is invariant under $S^{n}$ and $\phi_{i} \in \mathcal{M}$, it follows that

$$
\mathcal{M} \supseteq \sum_{i=1}^{r} \oplus \phi_{i} H^{p}\left(z^{n}\right) \oplus \mathcal{K}_{\mathcal{M}}^{\bar{\phi}}
$$

To establish the other containment, let $f \in \mathcal{M}$. Then as shown above, there exists an outer function $k \in H^{\infty}\left(z^{n}\right)$ such that $k f \in \mathcal{M} \cap L^{\infty}$. So we can write $k f=\sum_{i=1}^{r} \phi_{i} h_{i}+s$ for some $h_{i} \in H^{2}\left(z^{n}\right)$ and $s \in \mathcal{K}_{\mathcal{M} \cap L^{2}}^{\bar{\phi}}$. By using Lemma 3.8, we get

$$
n\left(\sum_{i=1}^{r}\left|h_{i}(z)\right|^{2}+\sum_{i=1}^{n}\left|s_{i}(z)\right|^{2}\right) \leq\left(\sum_{j=0}^{n-1}\left|k f\left(\alpha^{j} z\right)\right|\right)^{2}
$$

where $\alpha=e^{2 \pi i / n}$ and $s=\sum_{i=1}^{n} z^{i-1} s_{i}$ with $s_{i} \in L^{2}\left(z^{n}\right)$. Since $k \in H^{2}\left(z^{n}\right)$, we get

$$
\sum_{i=1}^{r}\left|\frac{h_{i}(z)}{k}\right|^{2}+\sum_{i=1}^{n}\left|\frac{s_{i}(z)}{k}\right|^{2} \leq \frac{\left(\sum_{j=0}^{n-1}\left|k f\left(\alpha^{j} z\right)\right|\right)^{2}}{n}
$$


This together with the very construction of $k$ shows that $h_{i} / k \in H^{p}\left(z^{n}\right)$ for all $i$, which further implies that $\sum_{i=1}^{r} \oplus \phi_{i} h_{i} / k \in \sum_{i=1}^{r} \oplus \phi_{i} H^{p}\left(z^{n}\right) \subseteq \mathcal{M}$.

Lastly, we need to show that $s / k \in \mathcal{K}_{\mathcal{M}}^{\bar{\phi}}$. It follows from the decomposition of $k f$ that $s / k \in \mathcal{M}$. By the definition of $s$,

$$
\sum_{j=1}^{n} \overline{\phi_{i j}} s_{j}=0
$$

for all $1 \leq i \leq r$. Observe that $s / k=\sum_{i=1}^{r} z^{i-1} s_{i} / k$ with $s_{i} / k \in L^{p}\left(z^{n}\right)$, and

$$
\sum_{j=1}^{n} \overline{\phi_{i j}} \frac{s_{j}}{k}=0
$$

for all $1 \leq i \leq r$. Thus, $s / k \in \mathcal{K}_{\mathcal{M}}^{\bar{\phi}}$ and the rest of the conclusions are now immediate using Case (1).

CASE (3). Finally, we assume that $p>2$. It can be verified that $\overline{\mathcal{M}}^{L^{2}}$ is a simply invariant subspace of $L^{2}$ under $S^{n}$, which further yields

$$
\overline{\mathcal{M}}^{L^{2}}=\sum_{i=1}^{r} \oplus \phi_{i} H^{2}\left(z^{n}\right) \oplus \mathcal{K}_{\overline{\mathcal{M}}^{L^{2}}}^{\bar{\phi}}
$$

where $\left\{\phi_{i}\right\}_{i=1}^{r}, r \leq n$, is a set of orthonormal vectors in $\overline{\mathcal{M}}^{L^{2}} \cap L^{\infty}$ such that $\phi_{i}$ 's do not vanish on sets of positive measure.

By virtue of Lemma 3.6, it is enough to show that

$$
\mathcal{M} \subseteq \sum_{i=1}^{r} \oplus \phi_{i} H^{p}\left(z^{n}\right) \oplus \mathcal{K}_{\mathcal{M}}^{\bar{\phi}} .
$$

Let $f \in \mathcal{M}$. Then there exist $h_{i} \in H^{2}\left(z^{n}\right)$ and $k \in \mathcal{K}_{\overline{\mathcal{M}}^{L^{2}}}^{\bar{\phi}}$ such that

$$
f=\sum_{i=1}^{r} \phi_{i} h_{i}+k .
$$

If we let $k=\sum_{j=1}^{n} z^{j-1} k_{j}, k_{j} \in L^{2}\left(z^{n}\right)$, then by using Lemma 3.8 we get

$$
\sum_{i=1}^{r}\left|h_{i}\right|^{2}+\sum_{i=1}^{n}\left|k_{i}\right|^{2} \leq \frac{\left(\sum_{j=0}^{n-1}\left|f\left(\alpha^{j} z\right)\right|\right)^{2}}{n} .
$$

This implies that

$$
\left|h_{i}\right| \leq \frac{\sum_{j=0}^{n-1}\left|f\left(\alpha^{j} z\right)\right|}{\sqrt{n}} \quad \text { and } \quad\left|k_{i}\right| \leq \frac{\sum_{j=0}^{n-1}\left|f\left(\alpha^{j} z\right)\right|}{\sqrt{n}} .
$$


Hence $h_{i} \in L^{p} \cap H^{2}\left(z^{n}\right)=H^{p}\left(z^{n}\right)$ and $k_{i} \in L^{p}\left(z^{n}\right)$ for all $i$. Thus, by Lemma 3.6 we deduce that $k \in \mathcal{K}_{\mathcal{M}}^{\bar{\phi}}$ and

$$
\mathcal{M}=\sum_{i=1}^{r} \oplus \phi_{i} H^{p}\left(z^{n}\right) \oplus \mathcal{K}_{\mathcal{M}}^{\bar{M}} .
$$

Again the other conclusions follow from Case (1).

4. Proof of Theorem B. In this section, we shall describe the simply and doubly invariant subspaces of $S^{n}$ which are contractively contained in $L^{p}$ for $1 \leq p \leq \infty$. But before we prove the theorem we give some preliminary results which might be of interest in their own right.

To prove the following result which describes the commutant of the operator $S^{n}$, we need to recall the operator $A_{\phi}$ defined in Section 2. If we let $\phi=\left(\phi_{i j}\right) \in M_{m n}\left(L^{\infty}\right)$ then $A_{\phi}$ is defined as

$$
A_{\psi}(f)=\sum_{i=1}^{m} z^{i-1}\left(\sum_{j=1}^{n} \psi_{i j} f_{j}\right)
$$

for every $f \in L^{2}$, where $f=\sum_{i=1}^{n} z^{i-1} f_{i}$ with $f_{i} \in L^{2}\left(z^{n}\right)$.

LEMma 4.1. The commutant $\left\{S^{n}\right\}^{\prime}$ of the operator $S^{n}$ is the set

$$
\left\{A_{\phi}: \phi=\left(\phi_{i j}\right) \in M_{n}\left(L^{\infty}\left(z^{n}\right)\right), 1 \leq i, j \leq n\right\} .
$$

Proof. The linear map $U: L^{2} \rightarrow L^{2}\left(z^{n}\right)$ defined by $U(z)=z^{n}$ is an onto isometry, and consequently the map

$$
V=U \oplus z U \oplus \cdots \oplus z^{n-1} U: \underbrace{L^{2} \oplus \cdots \oplus L^{2}}_{n \text { times }} \rightarrow L^{2}
$$

defines an onto isometry. Note that $V^{*} S^{n} V=\underbrace{S \oplus \cdots \oplus S}_{n \text { times }}$. Thus,

$$
\begin{aligned}
\left\{S^{n}\right\}^{\prime} & =\left\{V T V^{*}: T \in\{S \oplus \cdots \oplus S\}^{\prime}\right\} \\
& =\left\{V\left(M_{\psi_{i j}}\right) V^{*}:\left(\psi_{i j}\right) \in M_{n}\left(L^{\infty}\right)\right\} \\
& =\left\{\left(z^{i-j} U M_{\psi_{i j}} U^{*}\right):\left(\psi_{i j}\right) \in M_{n}\left(L^{\infty}\right)\right\} \\
& =\left\{\left(z^{i-j} M_{\phi_{i j}}\right):\left(\phi_{i j}\right) \in M_{n}\left(L^{\infty}\left(z^{n}\right)\right)\right\} .
\end{aligned}
$$

Finally, note that $A_{\phi}=\left(z^{i-j} M_{\phi_{i j}}\right)$ with respect to the decomposition $L^{2}=L^{2}\left(z^{n}\right) \oplus z L^{2}\left(z^{n}\right) \oplus \cdots \oplus z^{n-1} L^{2}\left(z^{n}\right)$.

We shall now obtain a characterization of the Hilbert spaces boundedly contained in $L^{q}, 1 \leq q \leq 2$, on which $S^{n}$ acts as a unitary. In view of the above lemma, the ideas of the proof of Theorem 2.1 in [PS1] and Theorem 2.3 in Red] can be extended to prove an appropriate generalization of both the results for $S^{n}$. For the sake of completeness we present the details here. 
Before we state and prove our result, we need another theorem stated in [Red, p. 3] and proved in [Mau.

THEOREM 4.2. If $\mathcal{H}$ is a Hilbert space and $T: \mathcal{H} \rightarrow L^{q}, 1 \leq q<2$, is a continuous linear operator, then there exist a continuous linear operator $U: \mathcal{H} \rightarrow L^{2}$ and $a g>0$ in $L^{2 q /(2-q)}$ such that $T=M_{g} U$, where $M_{g}$ is multiplication by $\mathrm{g}$.

ThEOREM 4.3. Let $\mathcal{H}$ be a Hilbert space boundedly contained in $L^{q}$, $1 \leq q \leq 2$. Then $S^{n}$ acts unitarily on $\mathcal{H}$ if and only if there exist a function $\mu$ in $L^{2 q /(2-q)}$ and $\phi=\left(\phi_{i j}\right) \in M_{n}\left(L^{\infty}\left(z^{n}\right)\right)$ such that $\mathcal{H}=\mathcal{R}\left(M_{\mu} A_{\phi}\right)$ isometrically, that is, $\|h\|_{\mathcal{H}}=\|h\|_{\mathcal{R}\left(M_{\mu} A_{\phi}\right)}$ for all $h$ in $\mathcal{H}$. When $\mathcal{H}$ is contractively contained in $L^{q}$, we have $\left\|M_{\mu} A_{\phi}\right\| \leq 1$.

Proof. If $\mathcal{H}=\mathcal{R}\left(M_{\mu} A_{\phi}\right)$ isometrically for some $\mu$ in $L^{2 q /(2-q)}$ and $\phi=$ $\left(\phi_{i j}\right) \in M_{n}\left(L^{\infty}\left(z^{n}\right)\right)$, then to prove that $S^{n}$ acts unitarily on $\mathcal{H}$, it is enough to note that $z^{n} h, z^{-n} h \in \mathcal{H}$ for all $h \in \mathcal{H}$.

To prove the converse, we assume that $S^{n}$ acts unitarily on $\mathcal{H}$ and let $C: \mathcal{H} \rightarrow L^{q}$ denote the bounded containment. We now divide the proof into two cases.

For $q=2$, there exists a unitary operator $U: \mathcal{H} \rightarrow \mathcal{H}$ such that $S^{n} C=$ $C U$. This means that $S^{n} C C^{*} S^{n *}=C C^{*}$, because $U$ is a unitary. Hence, by Lemma 4.1. $C C^{*}=A_{\psi}$ for some $\psi \in M_{n}\left(L^{\infty}\left(z^{n}\right)\right)$ and so by Douglas' factorization theorem $\mathcal{H}=\mathcal{R}(C)=\mathcal{R}\left(\left(A_{\psi}\right)^{1 / 2}\right)$. Note that $\left\{A_{\psi}: \psi \in\right.$ $\left.L^{\infty}\left(z^{n}\right)\right\}$ is a $C^{*}$-subalgebra of $M_{n}\left(L^{\infty}\right)$, thus there exists $\phi \in L^{\infty}\left(z^{n}\right)$ such that $A_{\psi}^{1 / 2}=A_{\phi}$. It can be easily verified that $\|h\|_{\mathcal{H}}=\|h\|_{\mathcal{R}\left(A_{\phi}\right)}$ and $\left\|A_{\phi}\right\| \leq 1$ whenever $\mathcal{H}$ is contractively contained in $L^{2}$.

We now proceed to the case $1 \leq q<2$. By Theorem 4.2 , there exist a $g>0$ in $L^{2 q /(2-q)}$ and a bounded operator $U: \mathcal{H} \rightarrow L^{2}$ such that $C=M_{g} U$. Thus, $\mathcal{H}=\mathcal{R}(C)=\mathcal{R}\left(M_{g} U\right)$, and since $g>0$ we see that $S^{n}$ acts unitarily on $\mathcal{R}(U)$. It then follows from the above case $(q=2)$ that $\mathcal{R}(U)=\mathcal{R}\left(A_{\phi}\right)$ isometrically for some $\phi \in M_{n}\left(L^{\infty}\left(z^{n}\right)\right)$ such that $U U^{*}=A_{\phi}^{2}$ with $A_{\phi} \geq 0$. Hence, $\mathcal{H}=\mathcal{R}\left(M_{g} A_{\phi}\right)$ isometrically, since $g>0$.

Note that

$$
\mu= \begin{cases}g & \text { when } 1 \leq q<2, \\ 1 & \text { when } q=2 .\end{cases}
$$

Finally, the estimate $\left\|M_{\mu} A_{\phi}\right\| \leq 1$ follows from the fact that $\|C\| \leq 1$, along with the observation that $C C^{*}=\left(M_{\mu} A_{\phi}\right)\left(M_{\mu} A_{\phi}\right)^{*}$.

REMARK 4.4. Note that the above theorem gives us an alternative characterization of the reducing part in the classical case of $L^{2}$.

As an immediate consequence we get the following analogue of Corollary 2.2 in [PS1] and Theorem 2.4 in [Red]. 
Corollary 4.5. Let $\mathcal{H}$ be boundedly contained in $L^{q}, 1 \leq q \leq 2$, and let $S^{n}$ act unitarily on $\mathcal{H}$. Then $\mathcal{H} \cap L^{2 q /(2-q)} \neq\{0\}$ if and only if $\mathcal{H} \neq\{0\}$.

The following result completes the characterization of Hilbert spaces boundedly contained in $L^{q}, 1 \leq q \leq \infty$, on which $S^{n}$ acts unitarily.

THEOREM 4.6. If $\mathcal{H}$ is a Hilbert space boundedly contained in $L^{q}, 2<$ $q \leq \infty$, on which $S^{n}$ acts unitarily, then $\mathcal{H}=\{0\}$.

Proof. If $\mathcal{H}$ is boundedly contained in $L^{q}$ for $q>2$, then $\mathcal{H}$ is boundedly contained in $L^{2}$, since $L^{q} \subseteq L^{2}$ and the $L^{q}$-norm dominates the $L^{2}$ norm. Thus by Theorem 4.3, $\mathcal{H}=\mathcal{R}\left(M_{\mu} A_{\phi}\right)$ for some function $\mu \in L^{\infty}$ and $\phi=\left(\phi_{i j}\right) \in M_{n}\left(L^{\infty}\left(z^{n}\right)\right)$. Then for each fixed $j$ with $1 \leq j \leq n$, the function $\sum_{i=1}^{n} z^{i-1} \phi_{i j}$ multiplies $L^{2}\left(z^{n}\right)$ into $\mathcal{H}$, and hence multiplies $L^{2}$ into $L^{q}$. However, no non-zero function in $L^{\infty}$ multiplies $L^{2}$ into $L^{q}$. Therefore, $\sum_{i=1}^{n} z^{i-1} \phi_{i j}=0$ for all $1 \leq j \leq n$. This implies that $\phi_{i j}=0$ for all $1 \leq i, j \leq n$ and hence $\mathcal{H}=\{0\}$.

We are now in a position to characterize the Hilbert spaces simply invariant under $S^{n}$ which are contractively contained in $L^{q}, 1 \leq q \leq \infty$. We begin with the following lemma.

Lemma 4.7. Let $\mathcal{M} \neq 0$ be a Hilbert space, simply invariant under $S^{n}$, contractively contained in $L^{q}, 1 \leq q \leq 2$, and on which $S^{n}$ acts isometrically. Let $\mathcal{N}=\mathcal{M} \ominus S^{n}(\mathcal{M})$. Then

(i) $\mathcal{N} \subseteq L^{2 q /(2-q)}$,

(ii) for every unit vector $\phi \in \mathcal{N},\|\phi f\|_{\mathcal{M}}=\|f\|_{2}$ for all $f \in H^{2}\left(z^{n}\right)$.

Moreover, if there exists a $p$ that satisfies $2 \leq p \leq 2 q /(2-q)$ and a $\delta>0$ such that

$$
\|f\|_{\mathcal{M}} \leq \delta\|f\|_{p} \quad \forall f \in \mathcal{M} \cap L^{p},
$$

then elements of $\mathcal{N}$ cannot vanish on sets of positive measure.

Proof. By using the Wold decomposition, we may write

$$
\mathcal{M}=\mathcal{M}_{1} \oplus \bigcap_{k \geq 0} S^{k n}(\mathcal{M})
$$

where $\mathcal{M}_{1}=\sum_{k=0}^{\infty} \oplus S^{k n}(\mathcal{N})$. In view of the hypothesis that $\mathcal{M}$ is simply invariant, we find that $\mathcal{M}_{1} \neq\{0\}$ and as a result, $\mathcal{N} \neq\{0\}$. If we choose any $\phi \in \mathcal{N}$ with $\|\phi\|_{\mathcal{M}}=1$, then $\left\{\phi z^{k n}\right\}_{k \geq 0}$ is an orthonormal sequence in $\mathcal{M}$.

Let $f \in H^{2}\left(z^{n}\right)$. Then $f=\sum_{k=0}^{\infty} \alpha_{k} z^{k n}$ and $f_{l}=\sum_{k=0}^{l} \alpha_{k} z^{k n}$ converges to $f$ in $L^{2}$. Note that

$$
\left\|\phi f_{l}\right\|_{\mathcal{M}}^{2}=\sum_{k=0}^{l}\left|\alpha_{k}\right|^{2}=\left\|f_{l}\right\|_{2}^{2},
$$

since $\left\{\phi z^{k n}\right\}_{k \geq 0}$ is an orthonormal set in $\mathcal{M}$. 
Thus, $\left\{\phi f_{l}\right\}_{l}$ is a Cauchy sequence in $\mathcal{M}$, which implies that there exists $g \in \mathcal{M}$ such that $\phi f_{l} \rightarrow g$ in $\mathcal{M}$, and so in $L^{q}$.

Consequently, there exists a subsequence $\left\{\phi f_{l_{j}}\right\}$ such that $\phi f_{l_{j}} \rightarrow g$ a.e. and $f_{l_{j}} \rightarrow f$ a.e. This yields $\phi f=g$ a.e. and hence $\phi f \in \mathcal{M}$. Thus, we conclude that $\phi H^{2}\left(z^{n}\right) \subseteq \mathcal{M} \subseteq L^{q}$, and hence $\phi \in L^{2 q /(2-q)}$. Also, $\|\phi f\|_{\mathcal{M}}=\|f\|_{2}$ for all $f \in H^{2}\left(z^{n}\right)$.

We now show that no element of $\mathcal{N}$ can vanish on a set of positive measure unless it is zero. Let $\phi \in \mathcal{N}$ and suppose it is zero on a set $A$ of positive measure. Then as in Lemma 3.1, we get an unbounded sequence $\left\{h_{l}\right\}$ in $H^{\infty}\left(z^{n}\right)$ such that the sequence $\left\{h_{l} \phi\right\}$ is in $\mathcal{M} \cap L^{\infty}$ and

$$
\left\|h_{l}\right\|_{2}=\left\|h_{l} \phi\right\|_{\mathcal{M}} \leq \delta\left\|h_{l} \phi\right\|_{p} .
$$

By the construction of $h_{l}$, the right hand side is bounded by a constant independent of $l$ and the left hand side is unbounded. This contradiction completes the proof.

Proof of Theorem $B$. We assume that $\mathcal{M}$ is contractively contained in $L^{q}$, $1 \leq q \leq 2$, and is boundedly contained in $L^{p}$ for some $p>2$, that is, $\|f\|_{\mathcal{M}} \leq \delta\|f\|_{p}$ for all $f \in \mathcal{M} \cap L^{p}$. It then follows from Lemma 4.7 that $\mathcal{M} \cap L^{p}$ is a non-zero closed subspace of $L^{p}$ and is simply invariant under $S^{n}$. Thus, by Case (3) of Theorem A, there exists an orthonormal set $\left\{\phi_{i}\right\}_{i=1}^{s}$ in $\left(\overline{\mathcal{M} \cap L^{p}} L^{2}\right) \ominus S^{n}\left(\overline{\mathcal{M} \cap L^{p}} L^{2}\right)$ such that $s \leq n$ and

$$
\mathcal{M} \cap L^{p}=\sum_{i=1}^{s} \oplus \phi_{i} H^{p}\left(z^{n}\right) \oplus \mathcal{K}_{\mathcal{M} \cap L^{p}}^{\bar{\phi}}
$$

where $\mathcal{K}_{\mathcal{M} \cap L^{p}}^{\bar{\phi}}=\bigcap_{k \geq 0} S^{k n}\left(\mathcal{M} \cap L^{p}\right)$.

Assume for the moment that $\left\{\phi_{i}\right\}_{i=1}^{s}$ is a basis of $\left(\mathcal{M} \cap L^{p}\right) / S^{n}\left(\mathcal{M} \cap L^{p}\right)$ so that $\operatorname{dim}\left(\left(\mathcal{M} \cap L^{p}\right) / S^{n}\left(\mathcal{M} \cap L^{p}\right)\right)=s$. Then we claim that the dimension of $\mathcal{N}=\mathcal{M} \ominus S^{n} \mathcal{M}$ is at most $s$. Towards a contradiction, suppose $\left\{\psi_{1}, \ldots, \psi_{s+1}\right\}$ is an orthonormal set in $\mathcal{N} \subseteq \mathcal{M}$. It follows from Lemma 4.7(i) that $\psi_{i}+S^{n}\left(\mathcal{M} \cap L^{p}\right) \in\left(\mathcal{M} \cap L^{p}\right) / S^{n}\left(\mathcal{M} \cap L^{p}\right)$ for all $i$.

Suppose there exist scalars $\alpha_{1}, \ldots, \alpha_{s+1}$ such that

$$
\sum_{i=1}^{s+1} \alpha_{i}\left(\psi_{i}+S^{n}\left(\mathcal{M} \cap L^{p}\right)\right)=0+S^{n}\left(\mathcal{M} \cap L^{p}\right) .
$$

This implies that $\alpha_{1} \psi_{1}+\cdots+\alpha_{s+1} \psi_{s+1} \in S^{n}\left(\mathcal{M} \cap L^{p}\right) \subseteq S^{n}(\mathcal{M})$. Since $\left\{\psi_{1}, \ldots, \psi_{s+1}\right\}$ is an orthonormal set in $\mathcal{N}$, it follows that $\alpha_{i}=0$ for all $i$. This proves that $\left\{\psi_{i}+S^{n}\left(\mathcal{M} \cap L^{p}\right)\right\}_{i=1}^{s+1}$ is a linearly independent set of $s+1$ elements in $\left(\mathcal{M} \cap L^{p}\right) / S^{n}\left(\mathcal{M} \cap L^{p}\right)$, which is a contradiction to the assumption. Thus, the dimension of $\mathcal{N}$ is at most $s$.

Finally, to show that $\left\{\phi_{i}\right\}_{i=1}^{s}$ is a basis of $\left(\mathcal{M} \cap L^{p}\right) / S^{n}\left(\mathcal{M} \cap L^{p}\right)$, we let $\sum_{i=1}^{s} \alpha_{i}\left(\phi_{i}+S^{n}\left(\mathcal{M} \cap L^{p}\right)\right)=0+S^{n}\left(\mathcal{M} \cap L^{p}\right)$. This implies that $\sum_{i=1}^{s} \alpha_{i} \phi_{i} \in$ 
$S^{n}\left(\mathcal{M} \cap L^{p}\right) \subseteq S^{n}\left(\overline{\mathcal{M} \cap L^{p}} L^{2}\right)$, which yields $\sum_{i=1}^{s} \alpha_{i} \phi_{i}=0$. Since $\left\{\phi_{i}\right\}_{i=1}^{s}$ is a linearly independent set, each $\alpha_{i}$ is zero. Thus, $\left\{\phi_{i}+S^{n}\left(\mathcal{M} \cap L^{p}\right)\right\}_{i=1}^{s}$ is a linearly independent set in $\left(\mathcal{M} \cap L^{p}\right) / S^{n}\left(\mathcal{M} \cap L^{p}\right)$.

Let $f \in \mathcal{M} \cap L^{p}$. Then $f=\sum_{i=1}^{s} \phi_{i} h_{i}+g$ for some $h_{i} \in H^{p}\left(z^{n}\right)$ and $g \in \mathcal{K}_{\mathcal{M} \cap L^{p}}^{\bar{\phi}}=\bigcap_{k \geq 0} S^{k n}\left(\mathcal{M} \cap L^{p}\right)$. Thus

$$
f+S^{n}\left(\mathcal{M} \cap L^{p}\right)=\sum_{i=1}^{s} \phi_{i} h_{i}+S^{n}\left(\mathcal{M} \cap L^{p}\right) .
$$

If we let $h_{i}=\sum_{k=0}^{\infty} \alpha_{k}^{i} z^{k n}$, then for each $i$ we get

$$
\phi_{i}\left(\sum_{k=0}^{l} \alpha_{k}^{i} z^{k n}\right) \rightarrow \phi_{i} h_{i} \quad \text { in } L^{p} \text { as } l \rightarrow \infty,
$$

since $\phi_{i} \in L^{\infty}$ for all $i$. Note that

$$
\phi_{i} h_{i}+S^{n}\left(\mathcal{M} \cap L^{p}\right)=\lim _{l} \alpha_{0}^{i}\left(\phi_{i}+S^{n}\left(\mathcal{M} \cap L^{p}\right)\right)=\alpha_{0}^{i}\left(\phi_{i}+S^{n}\left(\mathcal{M} \cap L^{p}\right)\right) .
$$

Thus, $f+S^{n}\left(\mathcal{M} \cap L^{p}\right)=\sum_{i=1}^{s} \alpha_{0}^{i} \phi_{i}+S^{n}\left(\mathcal{M} \cap L^{p}\right)$, and hence we conclude that $\left\{\phi_{i}+S^{n}\left(\mathcal{M} \cap L^{p}\right)\right\}_{i=1}^{s}$ is a basis of $\left(\mathcal{M} \cap L^{p}\right) / S^{n}\left(\mathcal{M} \cap L^{p}\right)$.

Let $\left\{\psi_{i}\right\}_{i=1}^{r}$ with $r \leq s$ be an orthonormal basis of $\mathcal{N}$. Then by using the Wold decomposition, we get

$$
\mathcal{M}=\sum_{i=1}^{r} \oplus \psi_{i} H^{2}\left(z^{n}\right) \oplus \bigcap_{k \geq 0} S^{k n}(\mathcal{M}) .
$$

It follows from Theorem 4.3 that $\bigcap_{k \geq 0} S^{k n}(\mathcal{M})=\mathcal{R}\left(M_{\mu} A_{\phi}\right)$ where $\mu$ and $\phi$ have the desired properties. Hence, we obtain the required decomposition of $\mathcal{M}$. For convenience, we skip to the last assertion in the theorem and prove it here. Note that $\bigcap_{k \geq 0} S^{k n}(\mathcal{M})$ is contractively contained in $L^{2}$ and $S^{n}$ acts as a unitary on it. If we now suppose $\bigcap_{k \geq 0} S^{k n}(\mathcal{M}) \neq\{0\}$, then by Corollary 4.5. $\bigcap_{k \geq 0} S^{k n}\left(\mathcal{M} \cap L^{2 q /(2-q)}\right) \neq\{0\}$, which further implies that $\bigcap_{k \geq 0} S^{k n}\left(\mathcal{M} \cap L^{p}\right) \neq\{0\}$. But this cannot happen unless $r<n$ by Case (3) of Theorem A. So, this proves that $\mathcal{R}\left(M_{\mu} A_{\phi}\right)=\{0\}$ if $r=n$.

To conclude the proof of this result, we need to prove (ii) and (iii). Note that (ii) immediately follows from Lemma 4.7. Thus, it only remains to establish (iii). For the sake of notational simplicity, we prove (iii) for $n=2$; the proof for general $n$ is identical. Fix an integer $i, 1 \leq i \leq n$, and let $\psi_{i}=\psi_{i 1}+z \psi_{i 2}$ where $\psi_{i j} \in L^{\infty}\left(z^{2}\right)$. We divide the proof for (iii) into the following cases.

CASE (1). Suppose $p=2$. Then the inequality

$$
\|h\|_{2}=\left\|\psi_{i} h\right\|_{\mathcal{M}} \leq \delta\left\|\psi_{i} h\right\|_{2}
$$


implies that

$$
\frac{1}{2 \pi} \int_{\mathbb{T}}\left(\delta^{2}\left(\left|\psi_{i 1}\right|+\left|\psi_{i 2}\right|\right)^{2}-1\right)|h|^{2} d m \geq 0
$$

for all trigonometric polynomials $h$, from which it follows that

$$
\left\|\left(\left|\psi_{i 1}\right|+\left|\psi_{i 2}\right|\right)^{-1}\right\|_{\infty} \leq \delta
$$

and hence $\left(\left|\psi_{i 1}\right|+\left|\psi_{i 2}\right|\right)^{-1} \in L^{\infty}$.

Case (2). We now assume that $2<p \leq 2 q /(2-q)$. For each positive integer $l$, we define

$$
E_{l}=\left\{z:\left|\psi_{i 1}(z)\right|>1 / l \text { or }\left|\psi_{i 2}(z)\right|>1 / l\right\}
$$

and for a fixed real $r$,

$$
k_{l}= \begin{cases}r \log \left(\left|\psi_{i 1}(z)\right|+\left|\psi_{i 2}(z)\right|\right) & \text { on } E_{l}, \\ 0 & \text { on } E_{l}^{c} .\end{cases}
$$

Then

$$
h_{l}=\exp \left(k_{l}+i \tilde{k}_{l}\right)
$$

belongs to $H^{\infty}\left(z^{2}\right)$ where $\tilde{k}_{l}$ denotes the harmonic conjugate of $k_{l}$.

Subcase $2($ a). Suppose $2<p \leq 2 q /(2-q)$ with $q<2$. In this case we obtain the inequality

$$
\begin{aligned}
\left(\frac{1}{2 \pi} \int_{E_{l}}\left(\left|\psi_{i 1}\right|+\left|\psi_{i 2}\right|\right)^{2 r} d m\right)^{1 / 2} & \leq\left\|h_{l}\right\|_{2}=\left\|\psi_{i} h_{l}\right\|_{\mathcal{M}} \leq \delta\left(\int_{\mathbb{T}}\left|\psi_{i}\right|^{p}\left|h_{l}\right|^{p} d m\right)^{1 / p} \\
& \leq \delta\left(\int_{\mathbb{T}}\left(\left|\psi_{i 1}\right|+\left|\psi_{i 2}\right|\right)^{p}\left|h_{l}\right|^{p} d m\right)^{1 / p} .
\end{aligned}
$$

Letting $l \rightarrow \infty$ and choosing $r=p /(2-p)<0$ yields

$$
\left(\frac{1}{2 \pi} \int_{\mathbb{T}}\left(\left|\psi_{i 1}\right|+\left|\psi_{i 2}\right|\right)^{-s} d m\right)^{1 / 2-1 / p} \leq \delta,
$$

that is, $\left(\left|\psi_{i 1}\right|+\left|\psi_{i 2}\right|\right)^{-1} \in L^{s}$, where $s=2 p /(p-2)$.

Subcase 2(b). Finally, we consider the case when $p=2 q /(2-q), q=2$. Here we choose $r=-1$. We find, upon taking the limits in the inequality

$$
\left(\frac{1}{2 \pi} \int_{E_{l}}\left(\left|\psi_{i 1}\right|+\left|\psi_{i 2}\right|\right)^{2 r} d m\right)^{1 / 2} \leq \delta\left\|\left(\left|\psi_{i 1}\right|+\left|\psi_{i 2}\right|\right) h_{l}\right\|_{\infty}
$$

and using the easily deduced fact $\lim _{l \rightarrow \infty}\left\|\left(\left|\psi_{i 1}\right|+\left|\psi_{i 2}\right|\right) h_{l}\right\|_{\infty}=1$, that

$$
\left(\left|\psi_{i 1}\right|+\left|\psi_{i 2}\right|\right)^{-1} \in L^{2} .
$$

This completes the proof of the theorem. 
The following result comes as a consequence of the above theorem; it asserts that, under the same conditions as in the theorem, there are no nontrivial simply invariant subspaces contractively contained in $L^{r}$ for $r>2$. We prove this by the same arguments used by Paulsen and Singh to prove [PS1, Corollary 5.2].

COROLlary 4.8. Let $\mathcal{M}$ be a simply invariant Hilbert space boundedly contained in $L^{r}$ for some $r>2$. Suppose that $S^{n}$ acts isometrically on $\mathcal{M}$ and there exist $2 \leq p \leq \infty$ and $\delta>0$ such that $\|f\|_{\mathcal{M}} \leq \delta\|f\|_{p}$ for all $f \in \mathcal{M} \cap L^{p}$. Then $\mathcal{M}=\{0\}$.

Proof. Assume that $\mathcal{M} \neq\{0\}$. Then $\mathcal{M}$ is a non-zero simply invariant Hilbert space contractively contained in $L^{2}$, since $L^{r} \subset L^{2}$. Also, it satisfies the condition as in the above theorem, by the hypotheses, and thus there exists $\phi \in L^{\infty}$ such that $\phi H^{2} \subseteq \mathcal{M}$. However, for each $\phi \in L^{\infty}$ there exists $f \in H^{2}$ such that $\phi f \notin L^{r}$, a contradiction.

Acknowledgements. We would like to thank the referee for careful reading and valuable comments. We would also like to thank Prof. Vern Paulsen for valuable discussions. The third author expresses appreciation for support of the Department of Mathematics, University of Houston, where part of this paper was written. He also acknowledges the facilities provided by the Mathematical Sciences Foundation.

\section{References}

[Beu] A. Beurling, Two problems concerning linear transformations in Hilbert space, Acta Math. 81 (1949), 239-255.

[BR] L. de Branges and J. Rovnyak, Square Summable Power Series, Holt, Rinehart and Winston, New York, 1966.

[DPRS] K. Davidson, V. Paulsen, M. Raghupathy and D. Singh, A constrained Nevanlinna-Pick interpolation problem, Indiana Univ. Math. J. 58 (2009), 709-732.

[Gam] T. Gamelin, Uniform Algebras, AMS Chelsea Publ., 2005.

[Hel1] H. Helson, Harmonic Analysis, Hindustan Book Agency, 1995.

[Hel2] - Lectures on Invariant Subspaces, Academic Press, New York, 1964.

[Hel3] - Analyticity on compact Abelian groups, in: Algebras in Analysis, Academic Press, 1975, 1-62.

[HL] H. Helson and D. Lowdenslager, Invariant subspaces, in: Proc. Internat. Sympos. Linear Spaces (Jerusalem, 1960), Jerusalem Academic Press, Jerusalem, and Pergamon, Oxford, 1961, 251-262.

[Hof1] K. Hoffman, Banach Spaces of Analytic Functions, Prentice-Hall, 1962.

[Hof2] -, Analytic functions and logmodular Banach algebras, Acta Math. 108 (1962), 271-317.

[Mau] B. Maurey, Théorèmes de factorisation pour les opérateurs linéaires à valeurs dans les espaces $L_{p}$, Astérisque 11 (1974).

[Ohn] Y. Ohno, Simply invariant subspaces, Tôhoku Math. J. 19 (1967), 368-378. 
[PS1] V. Paulsen and D. Singh, A Helson-Lowdenslager-de Branges theorem in $L^{2}$, Proc. Amer. Math. Soc. 129 (2001), 1097-1103.

[PS2] - - , Modules over subalgebras of the disk algebra, Indiana Univ. Math. J. 55 (2006), 1751-1766.

[RaRo] H. Radjavi and P. Rosenthal, Invariant Subspaces, 2nd ed., Dover Publ., 2003.

[Red] D. A. Redett, Sub-Lebesgue Hilbert spaces on the unit circle, Bull. London Math. Soc. 37 (2005), 793-800.

[RoRo] M. Rosenblum and J. Rovnyak, Hardy Classes and Operator Theory, Oxford Univ. Press, New York, 1985.

[San] M. Sand, Shift invariant spaces in $L^{2}$, Integral Equations Operator Theory 26 (1996), 210-221.

[Sar1] D. Sarason, Shift-invariant spaces from the Brangesian point of view, in: The Bieberbach Conjecture (West Lafayette, IN, 1985), Math. Surveys Monogr. 21, Amer. Math. Soc., Providence, RI, 1986, 153-166.

[Sar2] -, Sub-Hardy Hilbert Spaces in the Unit Disk, Univ. of Arkansas Lecture Notes Math. Sci. 10, Wiley, New York, 1994.

[Sin] D. Singh, Harmonic analysis on the unit circle: A personal perspective, Math. Student 76 (2007), 137-155.

[SS] D. Singh and U. N. Singh, On a theorem of de Branges, Indian J. Math. 33 (1991), 1-5.

[ST] D. Singh and V. Thukral, Multiplication by finite Blaschke factors on de Branges spaces, J. Operator Theory 37 (1996), 223-245.

[Sri] T. P. Srinivasan, Doubly Invariant Subspaces, Pacific J. Math. 14 (1964), 701707.

Sneh Lata, Meghna Mittal

Dinesh Singh

Department of Mathematics

Department of Mathematics

University of Houston

University of Delhi

Houston, TX 77204-3476, U.S.A.

Delhi 110007, India

E-mail: snehlata@math.uh.edu

mittal@math.uh.edu

E-mail: dineshsingh1@gmail.com

Received March 19, 2010

Revised version July 20, 2010

(6837) 